\title{
Radiotherapy with combined techniques of volumetric-modulated arc therapy (VMAT) and simultaneously intra-tumor inner escalated boost (SIEB) successfully managing a patient with locoregionally advanced maxillary sinus cancer: a case report
}

\author{
Li-Wen Huang ${ }^{1}$, Hon-Yi Lin ${ }^{1,2}$, Moon-Sing Lee ${ }^{1,2}$, Wen-Yen Chiou ${ }^{1,2}$, Liang-Cheng Chen ${ }^{1}$, \\ Chen-Lin Chi ${ }^{1}$, Shih-Kai Hung ${ }^{1,2}$ \\ ${ }^{1}$ Department of Radiation Oncology, Dalin Tzu Chi Hospital, Buddhist Tzu Chi Medical Foundation, Chia-Yi; ${ }^{2}$ School of Medicine, Tzu Chi \\ University, Hualien \\ Correspondence to: Shih-Kai Hung, MD, PHD. Department of Radiation Oncology, Dalin Tzu Chi Hospital, Buddhist Tzu Chi Medical Foundation, \\ No. 2, Min-Sheng Road, Dalin Town, Chia-Yi. Email: oncology158@yahoo.com.tw.
}

\begin{abstract}
A 46-year-old man had left nasal obstruction with abnormal blood-ting secretion for months and was diagnosed as having squamous cell carcinoma of left nasal cavity to left maxillary sinus, cT4a (anterior orbital content invasion; $6.9 \mathrm{~cm}$ ) N2bM0, stage IVA. Definitive concurrent chemoradiotherapy (CCRT) was planned due to his refusal of operation. In the present case, the simultaneously integrated inner-escalated boost (SIEB)-volumetric-modulated arc therapy (VMAT) technique was performed. By 25 fractions, the total dose of the total planning target volume $\left(\mathrm{PTV}_{\text {total }}\right)$-primary clinical target volume (CTVp)-primary gross target volume (GTVp) were 5,000-6,500-7,750 cGy, respectively. Equivalent dose in 2 Gy fractions

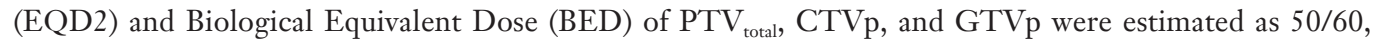
68.3/82, and 84.7/101.6 Gy, respectively. Additionally, in order to limit the normal tissue toxicities, the lower dose with 4,000 cGy in 25 fractions (fraction size of $160 \mathrm{cGy}$ ) was prescribed at the peripheral zone of the $\mathrm{PTV}_{\text {total }}$. During and after radiotherapy, no severe toxicities were noted (grade 3-4). At about one year after completion of CCRT, the gross tumor showed regression. Tumor excision was performed, and the pathological complete response status was noted. No recurrence and no new late toxicity had been noted in the 2 years of follow-up. The SIEB-VMAT technique is a safe and beneficial technique in treating enlarged head and neck tumor.
\end{abstract}

Keywords: Case report; simultaneously intra-tumor inner escalated boost (SIEB); maxillary sinus cancer

Received: 18 February 2020; Accepted: 08 April 2020; Published: 30 June 2020.

doi: $10.21037 /$ tro-19-46

View this article at: http://dx.doi.org/10.21037/tro-19-46

\section{Introduction}

Cancers of paranasal sinus are rare and comprise less than $1 \%$ of all cancers and about $3 \%$ to $5 \%$ of head and neck cancers (1). The maxillary sinus is the most prevalent cancer-arising site of primary paranasal sinuses.

Managing patients with maxillary sinus cancers remains a great challenge due to anatomical location in proximity to vital structures and significant diversity of histological types (2). More notably, maxillary sinus cancers are frequently locally advanced at presentation because they are usually asymptomatic for a long time. Even symptoms developed, early symptoms are similar to common nasal complaints that prevent from an alert for early diagnosis.

Because of its low prevalence, optimal treatment approach for locally advanced maxillary sinus carcinoma remains controversial. For patients with unresectable disease, definitive concurrent chemoradiotherapy (CCRT) may be a suitable treatment choice, demonstrating a 5 -year overall survival rate of $59.2 \%$, and disease-specific 
survival rate of $71.7 \%$ (3). According to extrapolations from results of a meta-analysis in patients with head and neck cancers (MACH-NC), when compared with RT alone, CCRT shows an absolute benefit of $6.5 \%$ on 5 -year overall survival (4).

CCRT provides as a treatment choice for managing patients with maxillary sinus cancers, but the best irradiating dose remains unclear. Dose escalation of radiotherapy (RT) is a subject of debate due to concerns of dose-related toxicities and uncertain survival benefits of dose-intensified prescription. Seemly, homogenous dose escalation may not be an optional choice because of a high local failure rate of $69 \%$ and potential harms for surrounding normal tissues (5).

Recently, combined RT techniques of volumetricmodulated arc therapy (VMAT) and simultaneously integrated inner-escalated boost (SIEB) (6) have been reported to gain double benefits in irradiating bulky tumors. First, it escalates RT dose per faction over central tumor zone for massively damaging cancer cells. Second, it maintains or slightly decreases fraction size over the peritumor zone for keeping a low risk of RT-related toxicities. By using this technique, it is possible to prescribe doses of BED more than 100 Gy to bulky tumors without gaining severe toxicities. However, whether applying VMAT-SIEB $\mathrm{RT}$ is really benefited for patients with locoregionally advanced maxillary sinus cancers is largely unknown.

Herein, we presented a patient with locoregionally advanced maxillary sinus cancer. He refused radical surgery, and definitive CCRT with combined VMATSIEB technique was conducted. Good tumor control (i.e., late pathological complete response) with a cost of limited toxicities was observed. We present the following case in accordance with the CARE reporting checklist (available at http://dx.doi.org/10.21037/tro-19-46).

\section{Case presentation}

A 46-year-old man with history of hypertension and hepatitis B virus infection for several years came to our hospital due to left nasal obstruction with abnormal bloodting secretion for months.

The physical examination showed hearing defect of left ear, nasal bleeding and post-nasal dripping on the admission day. On computed tomography (CT), enhanced masses were found in the left nasal cavity and left maxillary sinus with destruction of the anterior and medial wall of left maxillary sinus. The largest mass was $6.9 \mathrm{~cm}$ in diameter (Figure 1). Multiple enlarged lymph nodes (up to $1 \mathrm{~cm}$ in short axis) were observed over the left level II-III neck nodal basins. Biopsy was done, and histopathological examination showed squamous cell carcinoma of the left maxillary sinus, with tumor invasions to left nasal cavity and anterior orbital content, cT4aN2bM0, stage IVA (2015/11, AJCC 7e). After discussion with the patient and his families, definitive chemoradiotherapy (CCRT) was prescribed due to his refusal of surgery, which burdens a high risk of postoperation complications.

The patient was fixed with $\mathrm{U}$-frame mask in supine position. His arms and shoulders were pulled down and the hands were fixed in place under the buttocks. The CTsimulation ( $3 \mathrm{~mm}$ slice thickness) was performed with intravenous contrast.

Concurrent chemotherapy with two cycles of cisplatin $\left(100 \mathrm{mg} / \mathrm{m}^{2}, \mathrm{Q} 3 \mathrm{~W}\right)$ and RT with VMAT-SIEB technique were given (Figure 1B). The primary gross target volume (GTVp) and the nodal gross target volume (GTVn) included the primary gross tumor and the gross lymph nodes, respectively. The primary clinical target volume (CTVp) contained GTVp with 3-5 mm margin, left frontal sinus, left ethmoid sinus, left maxillary sinus, and sphenoid sinus. The nodal clinical target volume (CTVn) had GTVn with 3-5 $\mathrm{mm}$ margin, ipsilateral level I-III. The total planning target volume $\left(\mathrm{PTV}_{\text {total }}\right)$ were both CTVp and CTVn plus $3 \mathrm{~mm}$ margin for set-up error and intrafractional motion. PTV total $_{\text {, }}$ CTVp and GTVp were delivered with a dose gradient of 2, 3 and 3.5 Gy per fraction in the initial 5 fractions then shifted to 2, 2.5 and 3 Gy per fraction in the rest 20 fractions, respectively. Hence, by 25 fractions, total doses of PTV $\mathrm{Ptat}_{\text {tal }}$ CTVp, and GTVp were 50, 65, and 77.5 Gy, respectively (Figure 2B). Additionally, in order to limit normal tissue toxicities, a relatively low dose of $40 \mathrm{~Gy}$ in 25 fractions (i.e., a fraction size of $1.6 \mathrm{~Gy}$ ) was demarcated over the peripheral zone of the PTV $\mathrm{Ptal}_{\text {tal }}$ with $2 \mathrm{~mm}$ margin. No further boost was applied to the CTVn and GTVn. For having better accuracy, image guided RT was performed during the whole course of the treatment. $\mathrm{KV}$ cone beam computed tomography and KV 2-dimension images were performed once a week and the rest of the RT delivering day, respectively.

During and after RT, no severe toxicities (i.e., grade 3-4) were found. Only mild fatigue (grade 1) and moderate RT dermatitis (grade 2) were observed. One year after CCRT, the irradiated gross tumor showed significant tumor regression but still with residual tumor, being compatible with lasting partial response (Figure 1C). After re-discussion with the patient and his families, salvage surgery was done 

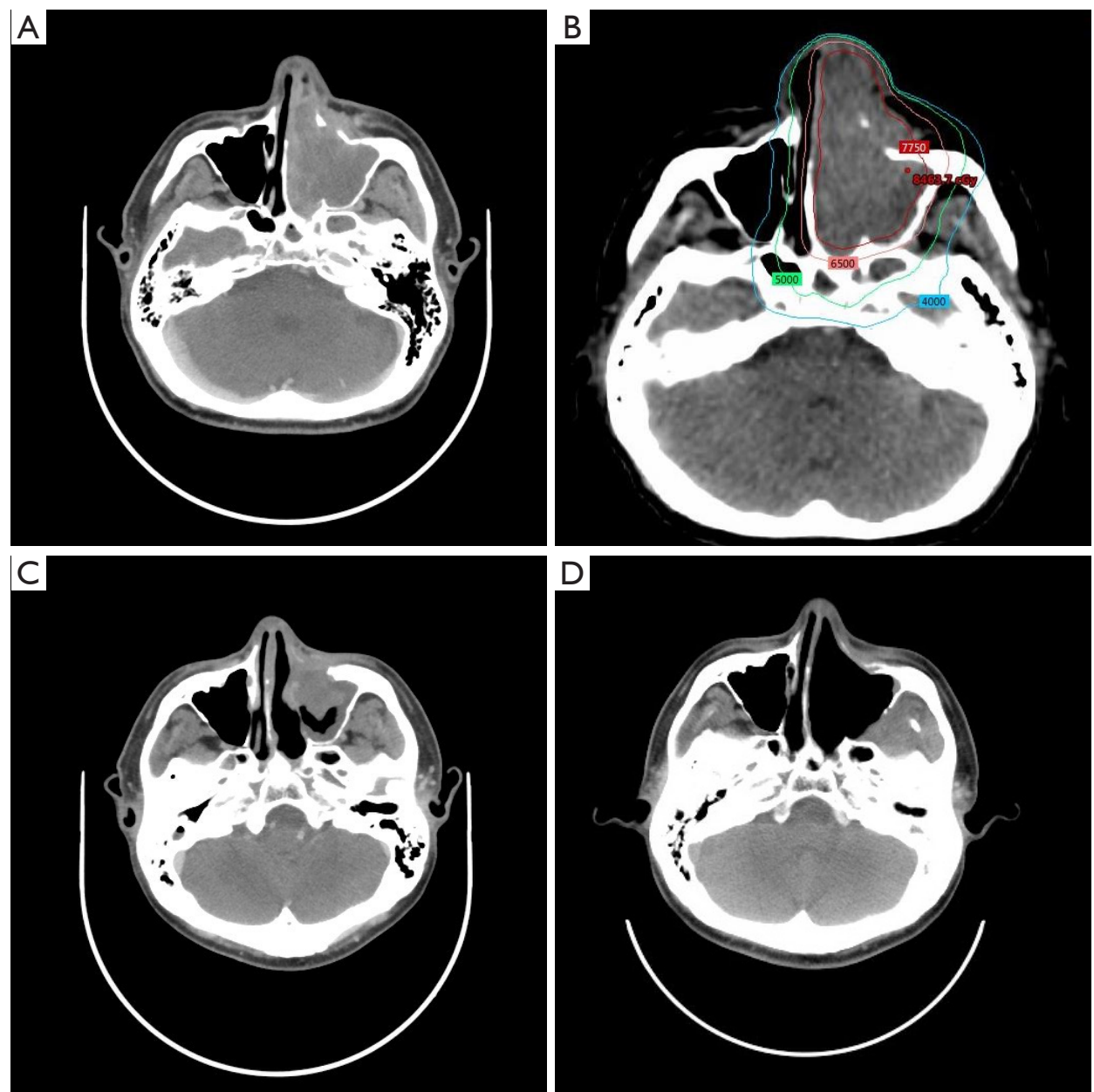

Figure 1 Sequence of CT image and dose distribution of treatment planning system. (A) The bulky tumor of the left maxillary sinus was measured about $6.9 \mathrm{~cm}$ in the largest diameter on CT image at the time of diagnosis. (B) RT with combined SIEB and VMAT technique was delivered with total doses of PTVtotal, CTVp, and GTVp were 50, 65, and 77.5 Gy in 25 fractions, generating a relatively protective low dose of 40 Gy at the peripheral zone of the PTVtotal. (C) The tumor showed significant regression but still residual tumor after one year of CCRT, being compatible with lasting partial response. (D) No residual tumor and recurrence were found after salvage surgery. CT, computed tomography; RT, radiotherapy; SIEB, simultaneously integrated inner escalated boost; VMAT, volumetric-modulated arc therapy; PTVtotal, total plan target volume; CTVp, primary clinical target volume; GTVp, primary gross target volume; CCRT, concurrent chemoradiotherapy.

(Figure 1D), and histopathological examination confirmed pathologically complete response (Figure 2). No cancer recurrence and obviously late RT toxicity were identified till the last follow-up (i.e., 2 years after CCRT).

The patient stated that he tolerated well during the whole course of the treatment and that no specific symptoms were noted during the following time.

\section{Discussion}

Maxillary sinus cancer is often unresectable due to the anatomical location in proximity of critical structures, i.e. cranial nerves and the orbit. For patients with locally advanced maxillary sinus cancer, CCRT may be a suitable treatment option. However, there is no large clinical trials focusing on locally advanced maxillary sinus cancer, and an optimal radiation dose is remaining unclear. Herein, we presented a patient with locoregionally advanced maxillary sinus cancer treated with CCRT of combined VMAT-SIEB technique with good clinical outcomes.

In the literature review, it is conflicting that RT dose escalation can improve local control and overall survival 

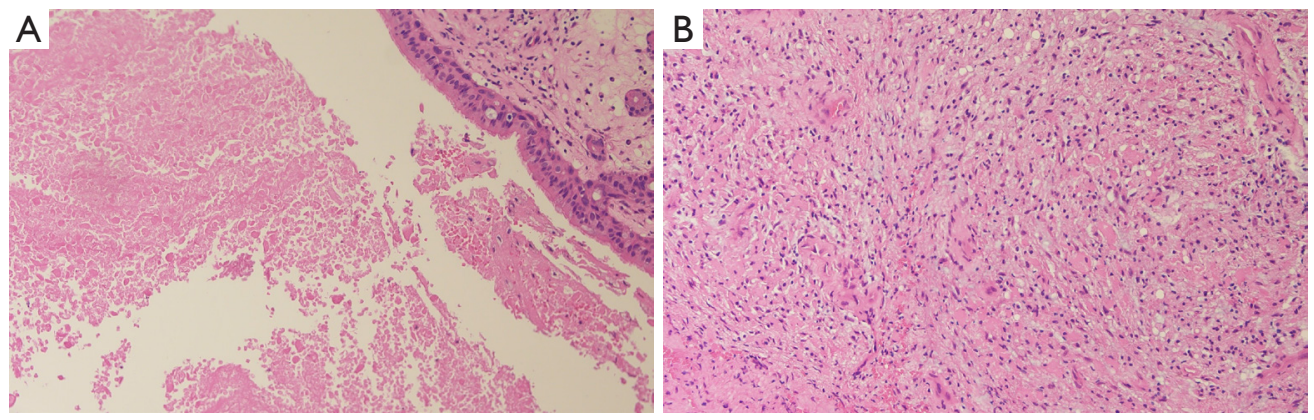

Figure 2 Post-salvage-surgery histopathological examination. (A) Nasal epithelium and necrotic materials (original magnification 20×20) are found. Only few inflammatory cells are observed in the necrotic materials. (B) Some viable tissue shows aggregates of foamy histiocytes and xanthogranuloma-like pattern (original magnification $20 \times 20$ ). All above histopathological slides show no evidence of residual cancer cells.

in cancers of the sinonasal cavity. Several studies have demonstrated no significant survival benefit of using doseescalated regimens when compared with conventional dose fractionation (5,7-10). Moreover, two retrospective studies $(5,10)$ showed RT with a total dose of more than 65 Gy demonstrates statistically significant worse overall and progress-free survival rates when compared with that of total dose below 65 Gy. However, on the contrary, Hoppe et al. gained reverse results (11). And this reverse effect was supported by another study showed that patients received RT dose of more than 60 Gy gains a clear benefit than that of those patients received dose of less than 60 Gy (12). These contradictory results may be related to that RT dose escalation may result in life-threatening radiationrelated toxicities due to proximity of critical structures. For overcoming this constraint, in the present case, we conducted RT with combined irradiating techniques of VMAT and SIEB.

For achieving a larger therapeutic gain, SIEB further modified irradiating technique from simultaneously integrated boost (SIB) (Figure 3). Previously, SIB is a commonly acknowledged irradiating technique that delivers different dose per fraction to separate target regions in the same number of RT fractions (13). For gaining better irradiation profiles, SIB mostly combined with intensitymodified radiotherapy (IMRT) (14) or VMAT (15). Generally, SIB delivers larger (e.g., 2.2-2.4 Gy), intermediate, and smaller (e.g.,1.6 Gy) doses per fraction for covering high-, intermediate-, and low-risk PTV. Note that dose distribution of SIB technique is homogeneous in each PTV.

For more safely and effectively irradiating bulky tumors that are adjacent to critical normal organs, modified SIB (i.e., SIEB) technique is developed (6,16-19). SIEB not only non-homogenously escalates dose within the high-risk PTV (e.g., from 2.2 to 3 Gy per fraction) but also attenuates dose to the peripheral zone of PTV (e.g., 1.2-1.6 Gy per fraction) (6,16-19). The GTVp and CTVp are both defined as high-risk PTV. The delivered dose is non-homogenously escalated from PTV to CTVp to GTVp, while the attenuated area is deescalated from PTV. To the authors' experience, GTVp, CTVp, PTV, and attenuated area (PTV with 1-2 mm margin) were usually irradiated with $150 \%$ to $160 \%, 120 \%$ to $130 \%, 100 \%$, and $80 \%$ to $90 \%$ of prescribed dose, respectively. As a result, SIEB further gains double benefits of increasing tumor control with a cost of limited toxicities.

Hence, in the present case, we applied CCRT with combined VMAT-SIEB technique to this non-surgical patient. Total doses of 50, 65, and 77.5 Gy were delivered in 25 fractions to PTV total, $\mathrm{CTV}$, and GTVp (Figure $1 B$ ). Equivalent dose in 2 Gy fractions (EQD2) and biological equivalent dose (BED) of $\mathrm{PTV}_{\text {total }}$, CTVp, and GTVp were estimated as 50/60,68.3/82, and 84.7/101.6 Gy, respectively. A protective low dose of 1.6 Gy per fraction was prescribed at the peripheral zone of the $\mathrm{PTV}_{\text {total }}$ for preventing unpredictable high dose over the normal tissues (e.g., the brain stem). No sever acute or late radiationrelated toxicities (i.e., grade 3-4) during the whole RT course and follow-up period. Surgical resection was done for salvaging the post-irradiating 1-year lasting residual tumor. Surprisingly, histopathological examination reported pathological complete response. The present case, in conjunction with the previously reported case (6), shed a light that combined RT techniques of VMAT and SIEB may be effectively to eradicate non-surgical bulky tumors that are adjacent to critical structures. Further prospective clinical trials are encouraged.

Definitive CCRT with combined VMAT and SIEB 


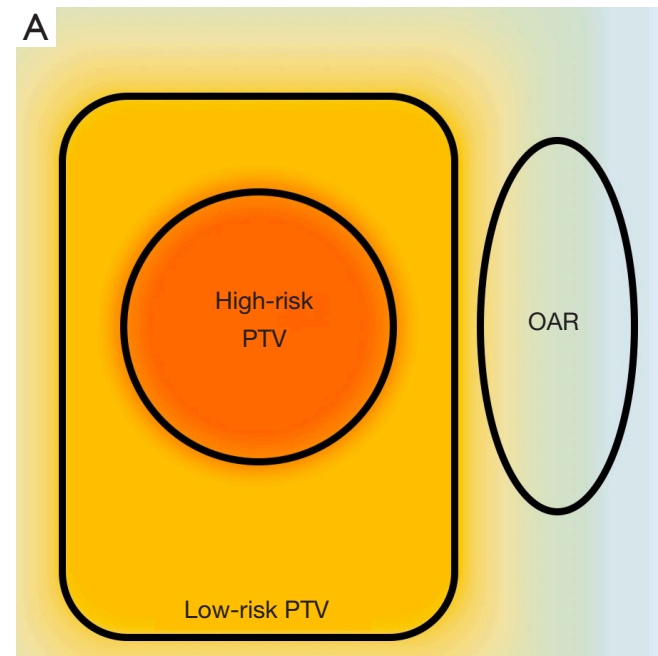

B

Figure 3 Cartoon figures for depicting dose-paintings of SIB and modified SIB (i.e., SIEB). (A) SIB simultaneously irradiates high-risk PTV (with a homologous higher dose per fraction, e.g., 2.4 Gy) and low-risk PTV (with a smaller fraction size, e.g., 1.6 Gy). The highrisk PTV is within or adjacent to the low-risk PTV. (B) Modified SIB (i.e., SIEB) not only integrates non-homologous inner dose escalation within high-risk PTV but also attenuates dose per fraction over the peripheral PTV zone near the organ at risk (OAR). As a result, SIEB further gains double benefits of increasing tumor control and decreasing irradiation toxicities. This radiotherapy technique is much useful in irradiating bulky tumors that are adjacent to critical normal organs. Several colors are used for representing dose-paintings, as follows: (I) red, intra-tumor inner escalated boost in SIEB (panel B); (II) orange, dose prescribed to high-risk PTV; (III) yellow, dose prescribed to lowrisk PTV; and, (IV) green, peri-PTV attenuated dose region in SIEB (panel B). SIB, simultaneously integrated boost; SIEB, simultaneously integrated inner escalated boost; PTV, planned target volume; OAR, organ at risk.

technique may be useful for managing locoregionally advanced maxillary sinus cancers with a cost of limited toxicities. However, there wasn't enough evidence to prove the benefits of SIEB technique. Further prospective trials are encouraged to demarcate clinical effective size of this type of combined RT techniques.

\section{Acknowledgments}

Funding: None.

\section{Footnote}

Reporting Checklist: The authors have completed the CARE reporting checklist. Available at http://dx.doi.org/10.21037/ tro-19-46

Conflicts of Interest: All authors have completed the ICMJE uniform disclosure form (available at http://dx.doi. org/10.21037/tro-19-46). SKH serves as an unpaid editorial board member of Therapeutic Radiology and Oncology from
Apr 2020 to Mar 2022. The other authors have no other conflicts of interest to declare.

Ethical Statement: The authors are accountable for all aspects of the work in ensuring that questions related to the accuracy or integrity of any part of the work are appropriately investigated and resolved. All procedures performed in studies involving human participants were in accordance with the ethical standards of the institutional and/or national research committee(s) and with the Helsinki Declaration (as revised in 2013). Written informed consent was obtained from the patient for publication of this Case report and any accompanying images. A copy of the written consent is available for review by the Editor-in-Chief of this journal.

Open Access Statement: This is an Open Access article distributed in accordance with the Creative Commons Attribution-NonCommercial-NoDerivs 4.0 International License (CC BY-NC-ND 4.0), which permits the noncommercial replication and distribution of the article with 
the strict proviso that no changes or edits are made and the original work is properly cited (including links to both the formal publication through the relevant DOI and the license). See: https://creativecommons.org/licenses/by-nc-nd/4.0/.

\section{References}

1. Muir CS, Nectoux J. Descriptive epidemiology of malignant neoplasms of nose, nasal cavities, middle ear and accessory sinuses. Clin Otolaryngol Allied Sci 1980;5:195-211.

2. Lewis JS Jr. Sinonasal Squamous Cell Carcinoma: A Review with Emphasis on Emerging Histologic Subtypes and the Role of Human Papillomavirus. Head Neck Pathol 2016;10:60-7.

3. Nishimura G, Tsukuda M, Mikami Y, et al. The efficacy and safety of concurrent chemoradiotherapy for maxillary sinus squamous cell carcinoma patients. Auris Nasus Larynx 2009;36:547-54.

4. Pignon JP, le Maître A, Maillard E, et al. Collaborative Group. Meta-analysis of chemotherapy in head and neck cancer (MACH-NC): an update on 93 randomized trials and 17,346 patients. Radiother Oncol 2009;92:4-14.

5. Chopra S, Kamdar DP, Cohen DS, et al. Outcomes of nonsurgical management of locally advanced carcinomas of the sinonasal cavity. Laryngoscope 2017;127:855-61.

6. Lin YH, Hung SK, Chiou WY, et al. Significant symptoms alleviation and tumor volume reduction after combined simultaneously integrated inner-escalated boost and volumetric-modulated arc radiotherapy in a patient with unresectable bulky hepatocellular carcinoma: A care-compliant case report. Medicine (Baltimore) 2016;95:e4717.

7. Waldron JN, O'Sullivan B, Warde P, et al. Ethmoid sinus cancer: twenty-nine cases managed with primary radiation therapy. Int J Radiat Oncol Biol Phys 1998;41:361-9.

8. Dirix P, Nuyts S, Geussens Y, et al. Malignancies of the nasal cavity and paranasal sinuses: long-term outcome with conventional or three-dimensional conformal radiotherapy. Int J Radiat Oncol Biol Phys 2007;69:1042-50.

9. Magrini SM, Nicolai P, Somensari A, et al. Which role for radiation therapy in ethmoid cancer? A retrospective analysis of 84 cases from a single institution. Tumori 2004;90:573-8.

10. Jansen EP, Keus RB, Hilgers FJ, et al. Does the combination of radiotherapy and debulking surgery favor survival in paranasal sinus carcinoma? Int J Radiat Oncol Biol Phys 2000;48:27-35.

11. Hoppe BS, Nelson CJ, Gomez DR, et al. Unresectable carcinoma of the paranasal sinuses: outcomes and toxicities. Int J Radiat Oncol Biol Phys 2008;72:763-9.

12. Szutkowski Z, Kawecki A, Wasilewska-Teśluk E, et al. Results of treatment in patients with paranasal sinus carcinoma. Analysis of prognostic factors. Otolaryngol Pol 2008;62:37-43.

13. Mohan R, Wu Q, Manning M, et al. Radiobiological considerations in the design of fractionation strategies for intensity-modulated radiation therapy of head and neck cancers. Int J Radiat Oncol Biol Phys 2000;46:619-30.

14. Spiotto MT, Weichselbaum RR. Comparison of 3D confromal radiotherapy and intensity modulated radiotherapy with or without simultaneous integrated boost during concurrent chemoradiation for locally advanced head and neck cancers. PLoS One 2014;9:e94456.

15. Franzese C, Fogliata A, Clerici E, et al. Toxicity profile and early clinical outcome for advanced head and neck cancer patients treated with simultaneous integrated boost and volumetric modulated arc therapy. Radiat Oncol 2015;10:224.

16. Nomiya T, Akamatsu H, Harada M, et al. Modified simultaneous integrated boost radiotherapy for an unresectable huge refractory pelvic tumor diagnosed as a rectal adenocarcinoma. World J Gastroenterol 2014;20:18480-6.

17. Nomiya T, Akamatsu H, Harada M, et al. Modified simultaneous integrated boost radiotherapy for large retroperitoneal malignant tumor: A case report. Oncol Lett 2015;9:2520-4.

18. Nomiya T, Akamatsu H, Harada M, et al. Modified simultaneous integrated boost radiotherapy for unresectable locally advanced breast cancer: preliminary results of a prospective clinical trial. Clin Breast Cancer 2015;15:161-7.

19. Crane $\mathrm{CH}$, Koay EJ. Solutions that enable ablative radiotherapy for large liver tumors: Fractionated dose painting, simultaneous integrated protection, motion management, and computed tomography image guidance. Cancer 2016;122:1974-86.

doi: $10.21037 /$ tro-19-46

Cite this article as: Huang LW, Lin HY, Lee MS, Chiou WY, Chen LC, Chi CL, Hung SK. Radiotherapy with combined techniques of volumetric-modulated arc therapy (VMAT) and simultaneously intra-tumor inner escalated boost (SIEB) successfully managing a patient with locoregionally advanced maxillary sinus cancer: a case report. Ther Radiol Oncol 2020;4:11. 